\title{
Oficinas Educativas e a Sistematização da Assistência de Enfermagem em Saúde Mental
}

\author{
Nathália Aparecida de Paula ${ }^{1}$ \\ Alda Martins Gonçalves ${ }^{2}$
}

\begin{abstract}
Apresentam-se, neste trabalho, os resultados da investigação sobre oficinas educativas direcionadas ao autocuidado, destacando-se a sua aplicabilidade na implementação da Sistematização da Assistência de Enfermagem em saúde mental. As oficinas fundamentaram-se nos princípios da Reforma Psiquiátrica e foram realizadas como parte da assistência de enfermagem em um serviço de atenção à saúde mental da criança e do adolescente. A investigação foi feita com base na análise dos registros das oficinas realizadas no período de abril de 2010 a novembro de 2011. Concluiu-se que as oficinas constituíram um espaço para intervenções de Enfermagem, promoção de saúde, correções do déficit de autocuidado e reabilitação psicossocial, além de contribuir, efetivamente, para o processo de Sistematização da Assistência de Enfermagem em saúde mental.
\end{abstract}

Descritores: Enfermagem Psiquiátrica; Assistência em Saúde Mental; Reforma dos Serviços de Saúde; Reabilitação.

\footnotetext{
${ }^{1}$ Mestranda, Escola de Enfermagem, Universidade Federal de Minas Gerais, Belo Horizonte, MG, Brasil.

2 PhD, Professor Adjunto, Escola de Enfermagem, Universidade Federal de Minas Gerais, Belo Horizonte, MG, Brasil.
}

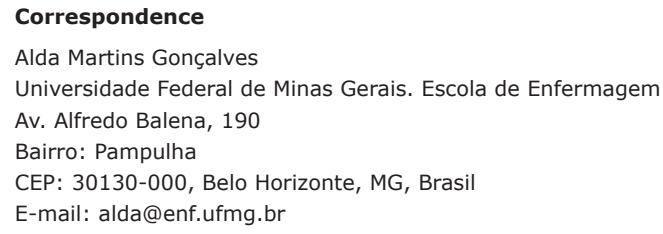




\section{Education Workshops and Nursing Care Systematization in Mental Health}

It presents the results of research on educational workshops focused on self-care, highlighting its applicability in the implementation of Nursing Care Systematization (NCS) in Mental Health. The workshops were based on the principles of Psychiatric Reform and were held as part of nursing care in a care service of children and adolescents Mental Health. The investigation was conducted by analyzing the workshop records held from April 2010 to November 2011. It was concluded that the workshops are a space for nursing interventions, health promotion, corrections of self-care deficit and psychosocial rehabilitation, and effectively contribute to the process of Systematization of Nursing Care in mental health.

Descriptors: Psychiatric Nursing; Mental Health Assistance; Health Care Reform; Rehabilitation.

\section{Oficinas Educativas y la Sistematización de la Asistencia de ENFERMERÍA EN SALUD MENTAL}

Se presenta los resultados de la averiguación sobre oficinas educativas dirigidas al autocuidado, destacando su aplicabilidad en la implementación de la Sistematización de la Asistencia de Enfermería (SAE) en Salud Mental. Las oficinas se fundamentaron en los principios de la Reforma Psiquiátrica y fueron realizadas como parte de la asistencia de enfermería en un servicio de atención a la Salud Mental del niño y del adolescente. La averiguación fue hecha del análisis de los registros de las oficinas realizadas en el período de abril de 2010 a noviembre de 2011. Se concluyó que las oficinas constituyeron en un espacio para intervenciones de enfermería, promoción de salud, correcciones del déficit de autocuidado y rehabilitación psicosocial, además de aportar, efectivamente, para el proceso de Sistematización de la Asistencia de Enfermería en salud mental.

Descriptores: Enfermería Psiquiátrica; Atención en Salud Mental; Reforma de la Atención de Salud; Rehabilitación.

\section{Introdução}

A psiquiatria vem, por longas décadas, modificando seu formato assistencial, cada vez mais considerando a importância da família e do meio sociocultural. Para o Ministério da Saúde, "embora contemporâneo da Reforma Sanitária, o processo de Reforma Psiquiátrica brasileira tem uma história própria, inscrita num contexto internacional de mudanças pela superação da violência

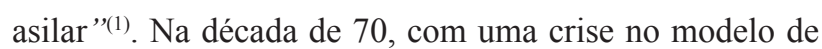
cuidado centrado na atenção hospitalar, relacionada aos movimentos sociais pelo direito dos usuários da saúde mental, no Brasil, teve início um processo com vistas a uma reforma da assistência psiquiátrica. Surge, em 1978, o Movimento dos Trabalhadores em Saúde Mental (MTSM), iniciativa fundamental para reformulação do campo da 
psiquiatria no país ${ }^{(2)}$. No ano 1987, o MTSM realizou, no Rio de Janeiro, um encontro de repercussão nacional com o lema "Por uma sociedade sem manicômios". Esse encontro influenciou diretamente na reestruturação da assistência prestada em saúde mental e impulsionou a realização da I Conferência Nacional de Saúde Mental. Nessa conferência discutiram-se formas de reorientar o modelo assistencial prestado em saúde mental ${ }^{(2)}$.

A realização da I Conferência Nacional de Saúde Mental, em desdobramento à $8^{\mathrm{a}}$ Conferência Nacional de Saúde, representa um marco histórico na psiquiatria brasileira, posto que reflete a aspiração de toda a comunidade científica da área, que entende que a política nacional de saúde mental necessita estar integrada à política nacional de desenvolvimento social do Governo Federal $^{(3)}$.

Foi nesse cenário que se deu a apresentação do projeto de lei do deputado Paulo Delgado, propondo regulamentar os direitos da pessoa com transtornos mentais e a gradual desativação dos manicômios ${ }^{(1)}$. Essa iniciativa foi considerada de grande importância no processo de legalização do movimento da Reforma Psiquiátrica. Em 1992, realizou-se a II Conferência Nacional de Saúde Mental.

A II Conferência Nacional de Saúde Mental tem como indicador de mudança a participação efetiva dos usuários na realização dos trabalhos de grupo, nas plenárias, nos debates e tribunas livres. Essa participação foi responsável pelo surgimento de uma nova dinâmica de organização do trabalho, por meio da qual os depoimentos pessoais, as intervenções culturais e a defesa dos direitos transformaram as relações e as trocas entre todos os participantes ${ }^{(4)}$.

Nos anos seguintes, várias iniciativas, práticas e movimentos em favor da Reforma Psiquiátrica tiveram continuidade. Em seis de abril de 2001, depois de onze anos de tramitação na Câmara dos Deputados e no Congresso Nacional do projeto Paulo Delgado, foi aprovada a Lei $\mathrm{n}^{\circ} 10.216$, que dispõe sobre a proteção e os direitos das pessoas portadoras de transtornos mentais e redireciona o modelo assistencial em saúde mental ${ }^{(5)}$.

Após a aprovação da Lei $n^{\circ} 10.216$, em dezembro do mesmo ano, foi realizada em Brasília a III Conferencia Nacional de Saúde Mental, com o tema "Cuidar sim, excluir não", considerada o marco zero para consolidação dos princípios que regem a Reforma Psiquiátrica no Brasil. "É no contexto da promulgação da Lei n”10.216 e da realização da III Conferência Nacional de Saúde Mental, que a política de saúde mental do governo federal, alinhada com as diretrizes da Reforma Psiquiátrica, passa a consolidar-se, ganhando maior sustentação e visibilidade"(1). Mudanças no campo da assistência em saúde mental, aliadas aos novos princípios preconizados pela Reforma Psiquiátrica brasileira, produziram a necessidade de reinventar e criar novas formas para cuidar e conviver com o doente mental ${ }^{(6)}$.

No intuito de consolidar essas mudanças na assistência e torná-la mais flexível e humanizada, os profissionais da saúde mental têm aperfeiçoado suas técnicas terapêuticas, por meio de fundamentos teóricos e práticos que permitem, além da participação dos usuários, reavaliar e desenvolver novas formas de cuidado. Dentre as várias propostas de mudanças na assistência psiquiátrica, atividades em grupos se multiplicaram e outros profissionais, além dos médicos, passaram a ter um papel terapêutico ${ }^{(7)}$. As práticas de cuidado prestadas pela Enfermagem Psiquiátrica, historicamente centradas na vigilância, restrição e contenção dos pacientes, passam a ser repensadas, exigindo fundamentação teórica, planejamento e organização de suas ações.

Acompanhando os movimentos de transformação da psiquiatria, transforma-se também a Enfermagem Psiquiátrica, cujos conceitos, mais influenciados pela Psiquiatria Social dão ênfase ao relacionamento com o paciente, individualmente, à manutenção do ambiente terapêutico e à ampliação do campo da Enfermagem para contato com paciente, família e comunidade ${ }^{(7)}$.

No final do século XX, procurando qualificar a assistência prestada por seus profissionais e contribuir com a Reforma Psiquiátrica, a Enfermagem expande a implantação da Sistematização da Assistência de Enfermagem (SAE) para os ambientes de cuidado em saúde mental. A SAE constitui um instrumento para favorecer o gerenciamento e a prestação do cuidado de Enfermagem. Sua aplicação sistemática exige conhecimentos específicos dos diagnósticos de Enfermagem, buscando produzir decisões sobre o que é necessário ser feito e quais ações e intervenções de Enfermagem contribuem, em cada caso, para se obter os resultados desejados: prevenção de doenças, promoção da saúde, tratamentos, alívio, conforto, reabilitação física e psicossocial ${ }^{(8)}$.

Por meio do projeto de extensão Sistematização da Assistência de Enfermagem em Saúde Mental, realizado no Centro Psíquico da Adolescência e Infância (CEPAI), elaboraram-se metodologias para a implantação e consolidação da SAE em saúde mental, nesse cenário. Esse projeto, aprovado sob número SIEX 400374, inserese nas atividades de ensino, pesquisa e extensão da Escola de Enfermagem da UFMG. O CEPAI é uma unidade da Rede de Saúde Mental pertencente à Fundação Hospitalar do Estado de Minas Gerais (FHEMIG). No CEPAI, espaço para assistência à criança e ao adolescente, o tratamento inclui a presença de um familiar, como acompanhante, durante o período que exige internação. Assim, promove a integração dos familiares ao tratamento da criança e do adolescente, amenizando o risco de exclusão social e atendendo o direito da convivência familiar previsto no Estatuto da Criança e do Adolescente, Lei n ${ }^{\circ} 8.069$ de 13 de julho de $1990^{(9)}$.

Durante o desenvolvimento das atividades do projeto Sistematização da Assistência de Enfermagem em Saúde Mental e do contato com os pacientes, acompanhantes e equipe de Enfermagem, foi evidenciada a necessidade de realizar uma ação complementar às atividades do projeto. Essa ação se deu no formato de oficinas educativas, com ênfase no autocuidado, fundamentadas nos princípios da Reforma Psiquiátrica. O presente trabalho teve por objetivo apresentar os resultados da investigação sobre a aplicação dessas oficinas educativas, destacando a sua 
aplicabilidade na implementação da Sistematização da Assistência de Enfermagem (SAE) em saúde mental.

\section{Oficinas Educativas}

A reprodução social da forma como são vistas as pessoas com transtornos mentais perpassa a prática clínica e constitui um processo complexo. Assim, a prática clínica exercida na rede de atendimento à saúde mental requer instrumentos e estratégias para a efetivação de ações resolutivas e de uma assistência que considere, de forma holística, cada ser humano. Nesse sentido, o desenvolvimento das abordagens terapêuticas no trabalho em saúde mental ocorre com vistas a melhorar o enfrentamento do transtorno psíquico, promovendo a reabilitação psicossocial do paciente ${ }^{(10-11)}$. Oficinas terapêuticas educativas em saúde mental são utilizadas como encontro entre pessoas em tratamento, seus acompanhantes e os profissionais de saúde, para promover cidadania e convivência com os diferentes, por meio da inclusão pela atividade ${ }^{(12)}$. As ações de Enfermagem para educação em saúde são momentos oportunos para se promover a integração entre o acompanhante, o paciente e a equipe de Enfermagem, além de ser um espaço de promoção e prevenção da saúde ${ }^{(13)}$.

A Portaria $\mathrm{n}^{\circ} 189$, de 19/11/1991, do Ministério da Saúde, determina que as práticas de oficinas nos serviços de saúde devem ser caracterizadas como atividades grupais de socialização, expressão e inserção social ${ }^{(14)}$. Contudo, para que alcancem os resultados esperados, faz-se necessário que as ações das oficinas educativas sejam planejadas, organizadas, baseadas em fundamentos teóricos, a partir do levantamento de demandas existentes, como é definido no processo de sistematização da assistência de Enfermagem.

Educação em saúde é qualquer combinação projetada de experiências de aprendizagem realizadas de forma a predispor, capacitar e reforçar o comportamento voluntário que promove a saúde dos indivíduos, grupos e comunidades. Por sua vez, promoção em saúde é uma combinação de apoios educacionais e ambientais que visam atingir ações e condições de vida conducentes à saúde ${ }^{(15-16)}$. Educação em saúde inclui estímulo ao autocuidado e correção de déficit, quando esse existe. "Quando um indivíduo é incapaz de cumprir seus requisitos de autocuidado, um déficit ocorre. É trabalho do enfermeiro determinar esses déficits e definir as modalidades de suporte"(17).

\section{Método}

Trata-se de investigação baseada na análise dos registros sobre o desenvolvimento das oficinas educativas elaboradas como uma ação complementar ao processo de implementação da SAE no CEPAI. As atividades das oficinas ocorreram no período de abril de 2010 a novembro de 2011, após discussão e aprovação do projeto pela equipe de profissionais da unidade.

As oficinas foram inseridas no cotidiano terapêutico da unidade e passaram a ser realizadas duas vezes por semana, em horário determinado. As atividades foram realizadas com crianças e adolescentes internados na unidade e contaram também com a participação dos familiares/ acompanhantes. Os registros das oficinas realizados em diário de campo constaram de anotações semanais das observações dos oficineiros sobre o desenvolvimento das atividades e suas intervenções terapêuticas no cotidiano dos participantes.

As oficinas basearam-se em ações colaborativas e participativas como princípio educativo para a promoção de saúde, reabilitação psicossocial e como espaço para intervenções e correções do déficit de autocuidado. Abordaram-se os seguintes temas: alimentação saudável, higiene bucal, obesidade, tabagismo, dependência química, estética e higiene pessoal, atividade física, relacionamento interpessoal, autoestima. Contemplaramse momentos de discussão e de reflexão e incluíram-se dinâmicas de grupo, atividades lúdicas, físicas e manuais. Para facilitar e intermediar o trabalho utilizaram-se como instrumentos e materiais: papel, tinta, pincéis, giz de cera, lápis de cor, canetas hidrocor, cartolina, emborrachado, retalhos de tecidos, balões, palito de picolé, revistas e jornais, tesouras, cola, música e outros.

Os principais objetivos das oficinas foram os de propiciar aos participantes o desenvolvimento de autonomia e visão crítica a respeito do autocuidado, envolvendo os profissionais da equipe de Enfermagem no cumprimento do papel de educador em saúde.

\section{Resultados}

A realização das oficinas educativas, usadas como método para a prática do cuidado, revelou-se estratégia valiosa para uma assistência prestada pelos profissionais de Enfermagem. Essas oficinas contribuíram efetivamente para a prestação da assistência de Enfermagem em saúde mental, de forma terapêutica, e sua sistematização na medida em que possibilitou o desenvolvimento da autonomia, da socialização e de visão crítica dos participantes a respeito do autocuidado. Combinaram promoção em saúde com apoios educacionais e ambientais para ações e condições de vida conducentes à saúde ${ }^{(15-16)}$. Envolveram os profissionais da equipe de Enfermagem no cumprimento do papel de educador em saúde, permitindo planejamento das atividades, organização do trabalho, levantamento de demandas e atendimento de necessidades dos participantes.

A operacionalização das oficinas educativas permitiu levantar demandas individuais e coletivas, possibilitando aos enfermeiros elaboração de diagnósticos precisos, bem como de planos terapêuticos adequados. Tais oficinas constituíram, portanto, uma metodologia para correção do déficit de autocuidado. Contemplaram momentos de discussão e de reflexão, constituindo um espaço para intervenções apropriadas a cada paciente e ao grupo. Pontua-se que as oficinas fortaleceram a organização do trabalho da Enfermagem em equipe, incentivaram a interação da equipe de Enfermagem com familiares/ acompanhantes e pacientes. Esse resultado reafirma a ideia de que oficinas terapêuticas educativas em saúde 
mental possam ser utilizadas como encontro entre pessoas em tratamento, seus acompanhantes e os profissionais de saúde, ao mesmo tempo em que promovem cidadania e convivência com os diferentes, por meio da inclusão pela atividade $^{(12)}$.

Durante a realização das oficinas, os participantes foram estimulados a desenvolver atividades de forma autônoma e independente. As discussões e reflexões favoreceram não só a prática do cuidar de si como, também, o trabalho em grupo, o respeito ao limite do outro e a elaboração de visão crítica em relação ao autocuidado, à autoestima e aos hábitos de vida saudáveis. Foram resgatados os conhecimentos prévios de cada participante acerca dos assuntos abordados, considerandose suas individualidades e seus contextos socioculturais, permitindo-lhes expressarem-se como sujeitos críticos, capazes de escolhas e de contribuição com o grupo. Facilitaram, assim, a reabilitação psicossocial dos sujeitos envolvidos.

A realização das oficinas possibilitou oferecer ao cliente a oportunidade, individual e de grupo, de discutir as experiências da doença e seus sentimentos com o enfermeiro para que ele pudesse criar um clima de confiança e apoio, prevenir recaídas e complicações, estimular a participação do cliente na experiência que vivencia, da forma mais independente possível. Ajudá-lo a se preparar para a reintegração social ${ }^{(12)}$.

As oficinas caracterizaram-se como dispositivos de cuidado para uma interação social saudável, durante o período da internação e para a vida em comunidade. Com isso, ajudaram a despertar desejo e coragem para superação de dificuldades relacionadas ao autocuidado, autoestima e interação social, fazendo os participantes sentirem que suas individualidades eram reconhecidas e valorizadas.

\section{Discussão}

Oficinas educativas fazem parte de uma lista das mais variadas estratégias terapêuticas que podem ser incluídas nas intervenções de Enfermagem, pois possibilitam o atendimento de demandas gerais ou específicas de um ou mais diagnósticos de Enfermagem definidos de acordo com a SAE.

É sempre bom lembrar que a atividade educativa não é um processo de condicionamento para que as pessoas aceitem, sem perguntar, as orientações que lhes são passadas. A simples informação ou divulgação ou transmissão de conhecimento, de como ter saúde ou evitar uma doença, por si só, não vai contribuir para que uma população seja mais sadia e nem é fator que possa contribuir para mudanças desejáveis no sentido da melhoria da qualidade de vida da população(18).

As diretrizes da Reforma Psiquiátrica lançaram grandes desafios aos profissionais da área que, necessariamente, têm que acompanhar a reestruturação da assistência prestada aos usuários da rede de saúde mental. Tais diretrizes exigem constante aperfeiçoamento das técnicas de cuidado, garantindo aos usuários autonomia e participação nas ações que envolvem o plano terapêutico. Os resultados desta investigação reafirmam a sistematização da assistência de Enfermagem em saúde mental como uma metodologia que garante organização e planejamento do cuidado, produzindo melhora na qualidade da assistência ${ }^{(7)}$. Essa metodologia corrobora os princípios e metas propostos pela Reforma Psiquiátrica, na medida em que direciona suas ações para melhor adaptação e recuperação do individuo quanto aos aspectos biopsicossociais. A reorganização na assistência em saúde mental reforça a necessidade de seus profissionais se capacitarem e aperfeiçoarem suas técnicas, garantindo a prestação de assistência de qualidade ${ }^{(19-20)}$. Para os profissionais de Enfermagem, torna-se essencial exercerem seu papel de educadores, como parte fundamental do planejamento, desenvolvimento e execução das estratégias terapêuticas.

\section{Conclusões}

Os resultados apresentados neste trabalho permitiram que se destacassem a pertinência do uso de oficinas educativas como estratégia metodológica importante para a aplicação da Sistematização da Assistência de Enfermagem em saúde mental, em cenários similares àquele em que foi desenvolvida. Os resultados necessariamente dependem da preparação, qualificação e envolvimento da equipe, com a utilização da metodologia de oficinas educativas.

Considera-se que a operacionalização de oficinas educativas foi uma experiência que favoreceu o aprendizado para o reconhecimento e elaboração de novas estratégias de cuidado e de educação em saúde na prática da Enfermagem. As atividades das oficinas oportunizaram aos envolvidos reflexão quanto ao cuidado prestado pela equipe de Enfermagem em saúde mental, tornandolhes possível conhecer e praticar inúmeras formas e possibilidades de prestar assistência de qualidade, de acordo com os fundamentos da Reforma Psiquiátrica.

Ressalta-se que este estudo aborda uma experiência local, não tendo, portanto, o propósito de generalizar os resultados. Entretanto, busca-se contribuir para futuros trabalhos ou experiências com objetivos e cenários semelhantes. Incentiva-se, ainda, a realização de novos estudos para o aprofundamento de discussões dos resultados do tema em análise.

\section{Referências}

1. Ministério da Saúde (BR). Reforma Psiquiátrica e política de Saúde Mental no Brasil. Conferência Regional de Reforma dos Serviços de Saúde Mental: 15 anos depois de Caracas. Brasília; 2005.

2. Costa JF. História da psiquiatria no Brasil. $5^{\circ} \mathrm{ed}$. Rio de Janeiro: Campus; 2002.

3. Ministério da Saúde (BR). Secretaria Nacional de Programas Especiais de Saúde. Centro de Documentação do Ministério da Saúde. I Conferência Nacional de Saúde Mental. Brasília; 1988.

4. Ministério da Saúde (BR). Secretaria de Assistência à Saúde. Departamento de Assistência e Promoção à Saúde. 
Coordenação de Saúde Mental. Relatório final da $2^{\mathrm{a}}$ Conferência Nacional de Saúde Mental. Brasília; 1994.

5. Lei no 10.216, de 6 de abril de 2001 (BR). Dispõe sobre a proteção e os direitos das pessoas portadoras de transtornos mentais e redireciona o modelo assistencial em saúde mental. Brasília, 6 de abril de 2001. $180^{\circ}$ da Independência e $113^{\circ}$ da República.

6. Barros S, Oliveira MAF, Silva ALA. Práticas inovadoras para o cuidado em saúde. Rev Esc Enferm USP. [Internet]. 2007 [acesso $2 \mathrm{dez}$ 2011]. Disponível em: http:/www.scielo.br/scielo.php?pid=S008062342007000500013\&script $=$ sci_arttext

7. Rocha RM. Enfermagem Psiquiátrica: Que papel é este?. Instituto Franco Basaglia. Rio de Janeiro: TeCorá; 1994.

8. Lopes FL, Szewczyk MSC, Lunardi VL, Santos SSC. SAE como um novo fazer na atividade da enfermeira com base na complexidade de Edgar Morin. Cogitare Enferm. [Internet]. 12(1); 2007 [acesso 13 jan 2013]. Disponível em: http://ojs. c3sl.ufpr.br/ojs/index.php/cogitare/issue/view/585.

9. Ministério da Saúde (BR). Lei n. 8069/90. Estatuto da Criança e do Adolescente. Dispõe sobre o Estatuto da Criança e do Adolescente e dá outras providências. Brasília; 2000.

10. Amarante P. Saúde mental e Atenção Psicossocial. Temas em Saúde. Rio de Janeiro: Fiocruz; 2007.

11. Oliveira AGB, Ataíde IFC, Silva MA. A invisibilidade dos problemas de saúde mental na atenção primária: o trabalho da enfermeira construindo caminhos junto às equipes de saúde da família. Texto Contexto Enferm. 2004;13:618-24.

12. Valladares ACA, Lappann-Botti NC, Mello R, Kantorski LP, Scatena MCM. Reabilitação Psicossocial através das Oficinas Terapêuticas e/ou Cooperativas Sociais. Rev Eletr Enferm. [Internet]. 2003. [acesso 12 set 2011]. Disponivel em: www.fnpu.br/revista.

13. Stefanelli MC, Fukuda IMK, Arantes EC. Enfermagem Psiquiátrica: em suas dimensões assistenciais. Barueri: Manole; 2008.

14. Ministério da Saúde (BR). Secretaria de Assistência à Saúde. Portaria n ${ }^{\circ} 189$ de 19/11/199. D.O.U. de 11/12/1991 e Portaria $n^{\circ} 224$ de 29/01/1992. D.O.U. de 30/01/1992. Brasília; 1991.

15. Candeias NMF. Conceitos de educação e de promoção em saúde: mudanças individuais e mudanças organizacionais. Rev Saúde Pública. 1997;31(2):209-13.

16. Botti NCL, Cotta EM, Achoa C. Oficina bem viver construção de tecnologias e significados de educação em saúde na área da saúde mental. SMAD, Rev. Eletrônica Saúde Mental Álcool Drogas (Ed. port.) [Internet]. 6(esp):471-92; 2010 [acesso $27 \mathrm{dez}$ 2012]. Disponível em: http://pepsic. bvsalud.org/scielo.php?script=sci_arttext\&pid=S180669762010000300010\&lng=pt\&nrm=iso

17. Orem ED. Nursing: concepts of practice. New York: McGraw-Hill; 1971.

18. Secretaria de Estado da Saúde de São Paulo (BR). Educação em Saúde. Planejando as Ações educativas: Teoria e Prática. Manual de operacionalização das ações educativas no SUS. [Internet]. São Paulo; 2001. [acesso 20 out 2011]. Disponível em: ftp://ftp. cve.saude.sp.gov. br/doc_tec/educacao.pdf
19. Candido MCFS, Furegato ARF. Atenção da enfermagem ao portador de transtorno depressivo: uma reflexão. SMAD, Rev. Eletrônica Saúde Mental Álcool Drogas (Ed. port.). [Internet]. 1(2); 2005 [acesso 25 dez 2012]. Disponível em: $\quad$ http://pepsic.bvsalud.org/scielo.php?script=sci arttext\&pid=S1806-69762005000200008\&lng=pt\&nrm=iso 20. Lorenzi JL, Oliveira MAF, Silva ALA. Sistematização da Assistência de Enfermagem em Saúde Mental no Município de Cascavel: Proposta de Trabalho. $2^{\circ}$ Seminário Nacional Estado e Políticas Sociais no Brasil. Cascavel; 2005. 